\title{
RETRACTED ARTICLE: The value of U-NGAL expression as a potential prognostic biomarker in patients with renal cancer after neoadjuvant chemotherapy with cisplatin
}

\author{
Tahereh Ebrahimi ${ }^{1}$ - Hamid Pirasteh ${ }^{2}$ Bijan Rezakhaniha ${ }^{3}$ - Banafshe Dormanesh ${ }^{4}$. \\ Rahman Ghaffarzadegan Rabati ${ }^{5}$ Emad Yahaghi $^{6} \cdot$ Aram Mokarizadeh $^{7}$
}

Published online: 25 June 2015

(C) International Society of Oncology and BioMarkers (ISOBM) 2016

\section{RETRACTED ARTICLE: Tumor Biol.}

DOI 10.1007/s13277-015-3680-z

This article has been retracted at the request of the Editorin-Chief, the International Society of Oncology and BioMarkers (ISOBM) and the Publisher per the Committee on Publication Ethics guidelines. The article shows evidence of irregularities in authorship during the submission process, there is strong reason to believe that the peer review process was compromised and irregularities were found in the Materials and methods section:

"Ten clinically mature men (25-46 years old)" found in the "Groups and CP administration" versus "Ranges of NGAL

Electronic supplementary material The online version of this article (doi:10.1007/s13277-015-3680-z) contains supplementary material, which is available to authorized users.

Aram Mokarizadeh

a.mokarizadeh@muk.ac.ir

1 Genetic and Agricultural Biotechnology, Institute of Tabarestan, Mazandaran, Iran

2 Department of Nephrology, AJA University of Medical Sciences, Tehran, Iran

3 Department of Urology, Imam Reza Hospital, AJA University of Medical Sciences, Tehran, Iran

4 Department of Pediatric Nephrology, AJA University of Medical Sciences, Tehran, Iran

5 Shahid Abbas Abdollahi, Molecular Biology Research Center of Shahid Mahallati Hospital, Tabriz, Iran

6 Baqiyatallah University of Medical Sciences, Tehran, Iran

7 Cellular \& Molecular Research Center, Kurdistan University of Medical Sciences, Sanandaj, Iran elevated in canine following CP injection" found in Nephrotoxicity biomarker in kidney tissue.

"Materials and methods

Groups and $\mathrm{CP}$ administration

A total of ten clinically mature men (25-46 years old) were obtained from private clinics, Tehran, Iran. The patients were habituated to their designated dwelling for at least 7 days before the first day of dosing. The patients were assigned into two groups, control group $(n=3)$ and test group $(\mathrm{n}=7)$, by an agreed randomization scheme planned to arrive like category average body weights. CP (Mylan Pharmaceutical Co, Greece) was administered intravenously at $50 \mathrm{mg} / \mathrm{m} 2$ for five consecutive days [22]."

"Materials and methods

Nephrotoxicity biomarker in kidney tissue

Ranges of NGAL elevated in canine following CP injection despite the infusion of high values of hydration and contrasted to baseline, serum, and urinary biomarker concentrations were notably elevated at 28 days (Figs. 1, 2, and 3)."

As such the validity of the content of this article cannot be verified.

The online version of this article contains the full text of the retracted article as electronic supplementary material. 